\title{
Design and Analysis of the AINiCo Hybrid Magnet in EMS Maglev Vehicles
}

\author{
LV Chao and LI Jie \\ College of Mechatronics Engineering and Automation, National University of Defense Technology, Changsha, Hunan 410073, China
}

\begin{abstract}
In order to solve the problem of hybrid electromagnet lock orbit, we design a new type of AlNiCo-NdFeB hybrid levitation electromagnet. The theoretical analysis has be carried on and mathematical model is established for AlNiCo-NdFeB hybrid levitation electromagnet. Through two dimensional simulation, the electromagnetic characteristics of the suspended electromagnet are analyzed in the 3 typical operating conditions, which are in heavy load at gap $8 \mathrm{~mm}$, in full load at gap $16 \mathrm{~mm}$ and in no-load at gap $3 \mathrm{~mm}$. And it's compared with the traditional electromagnetic magnet and $\mathrm{NdFeB}$ hybrid electromagnet. Calculation and analysis show that the new hybrid levitation electromagnet can effectively solve the problems of the electromagnet lock orbit, at the same time, have a good dynamic performance and suspension regulation performance.
\end{abstract}

\section{Introduction}

EMS maglev train suspended on the track in a given gap by the attraction of the electromagnet, with the advantages of small bending radius, strong gradeability, low noise, comfort and safety, etc. It gained more and more attention and favor, and it has been hailed as urban internal and inter-city new traffic system in the $21 \mathrm{st}$ century $[1,2]$.

The EMS maglev train electromagnet species are the electromagnetic suspension, electromagnetic-permanent magnet hybrid levitation, superconducting suspension, etc [3]. The representation of the EMS maglev train is the HSST maglev train in Japan and TR08 maglev train in Germany. They have adopted conventional electromagnetic suspension electromagnet structure, matured in technology, realized commercial operation respectively in Japan and shanghai china [4]. And conventional electromagnetic levitation maglev trains provided all electromagnet levitation force, in order to meet the design requirements of bearing capacity, witch need large suspended current, and that lead to the coil current density sharp fever beyond its critical value.Under the condition of full load, although the conventional electromagnetic levitation electromagnetic ferromagnetic are unsaturated, saturated. But due to the current saturation, carrying capacity cannot be improved.

To solve the defects existed in the conventional electromagnetic, the new suspension system was designed. The permanent magnet was added to the electromagnet, then electromagnetic permanent magnet hybrid suspension system formed. Under the condition that the carrying capacity is not reduced, the suspension current is reduced, and the current density of the coil is controlled in the range of no sharp heating of the conductor. At present, the electromagnetic permanent magnet hybrid electromagnet structure are series the permanent magnets in the magnetic yoke, series coil magnetic potential [5]. After adding permanent magnets, suspended current decreased, and suspended power consumption decreased. Coil winding of the current density can be greatly reduced, the coil heating problems are solved, and suspended sensor working environment is improved.

However, the existing electromagnetic permanent magnet hybrid suspension system has inherent defects. It usually adopts zero current suspension. That is to say, in the equilibrium position, electromagnet provides most of the levitation force. The square of the electromagnetic force of permanent magnet and suspension gap is inverse proportion. So in the case of levitation gap smaller, suction increases quickly, easy to lose control, adsorption on the orbit, cause harm to the train.

Take the advantage of energy-saving of electromagnetic permanent magnet hybrid system, at the same time, improv the ability to prevent the adsorption orbit. To improve the existing electromagnetic permanent magnet hybrid electromagnet structure. The two kind of permanent magnet $\mathrm{NdFeB}$ and $\mathrm{AlNiCo}$ are seried into the yoke, instead of traditional permanent magnet part of hybrid electromagnet. $\mathrm{NdFeB}$ permanent magnet is used as the permanent magnetic circuit, which is the main source of levitation force, clinging to reduce power consumption. At the same time, AlNiCo permanent magnet is used as the reversible magnetic circuit, while the electromagnetic coil is designed around the alnico magnets, constitute the modules to prevent lock orbit.

In a new hybrid levitation electromagnet, when loading a positive dc pulse, AlNiCo magnets was magnetized, produced the same pole direction as the 
$\mathrm{NdFeB}$ magnetic, thus magnet magnetic force is strengthened; When loading reverse dc pulse, AlNiCo magnets was reverse magnetized, so magnet magnetic force reductions and suction less than body gravity, thus the body fall down from orbit, achieve the function of prevent lock orbit.

This article mainly carries on the finite element electromagnetic simulation, and contrastive analysis with the electromagnetic magnet, traditional electromagnetic permanent magnet hybrid electromagnet. Demonstrate the performance and feasibility of it [6]. For applied in maglev train, lay the foundation for the appliment of new electromagnetic permanent magnet hybrid levitation electromagnet structure, which have abilities of prevent lock orbit and good dynamic performance, in maglev train.

\section{New hybrid electromagnet structure model}

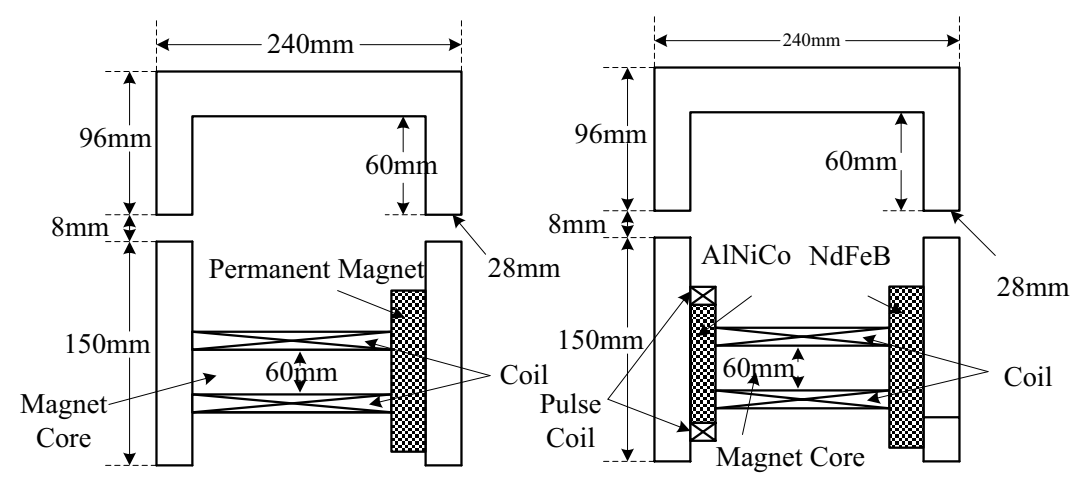

(a)
Compared with the traditional hybrid electromagnet, on the basis of the original structure, this new hybrid levitation electromagnet series AlNiCo permanent magnet in the yoke. The 3 kinds of electromagnet coil has 360 turns. Materials of new hybrid electromagnet is the same as traditional electromagnet. Specific size is shown in Fig. 1 . In (a), the thickness of NdFeB is $26 \mathrm{~mm}$, and it' s height is $90 \mathrm{~mm}$. In (b), the thickness of NdFeB is 26 $\mathrm{mm}$, and it's height is $90 \mathrm{~mm}$; the thickness of AlNiCo is $26 \mathrm{~mm}$, and it's height is $90 \mathrm{~mm}$. Orbital size design adopted with existing rail $F$ the corresponding specifications.

As shown in Fig. 1, from left to right, followed by $\mathrm{NdFeB}$ magnet, AlNiCo $\mathrm{NdFeB}$ magnet blend, electromagnetic magnet. The grey part of the figure is $\mathrm{NdFeB}$ permanent magnets. Upper to orbit, the white part of the lower is permeability core.

\section{Calculation of electromagnetic force}

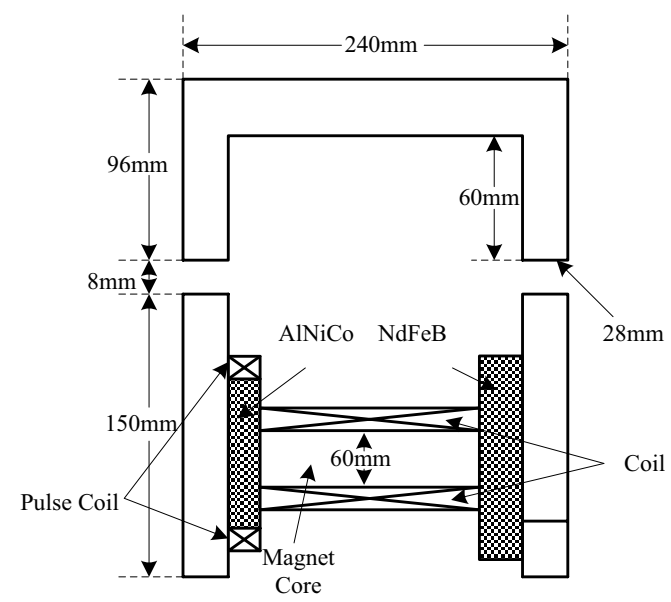

Figure 2. AlNiCo hybrid electromagnet structure model.

By the ampere loop theorem:

$$
\oint H . d l=N I
$$

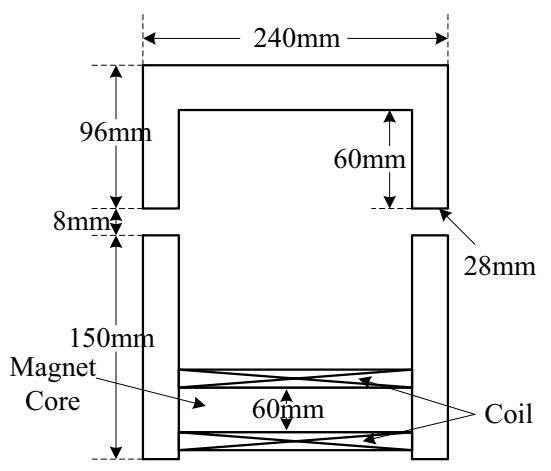

(c)
Figure 1. 3 kinds of electromagnet structure model.

$$
H=N I / l
$$

Among them, $\mathrm{N}$ is defined as the coil number of turns, I is defined as the coil current. Set core of absolute permeability as, thus the magnetic induction intensity :

$$
B=\mu H=\frac{\mu N I}{l}
$$

The core flux:

$$
\phi=B S=\frac{\mu N I S}{l}
$$

Magnetic circuit magnetic potential:

$$
E m=\oint H \cdot d l=N I
$$

By the magnetic circuit ohm's law can be core magnetic resistance of magnetic circuit. 


$$
R m=\frac{E m}{\phi}=\frac{l}{\mu S}
$$

The magnetic circuit equation is established:

$$
R_{f e} \phi+R_{c o} \phi+R_{p m} \phi+R_{\text {air }} \phi=N I+E_{p m}+E_{c o}
$$

Thus:

$$
\begin{gathered}
\phi=\frac{N I+E_{c o}+E_{p m}}{R_{\text {air }}+R_{f e}+R_{c o}+R_{p m}} \\
B=\frac{\phi}{A}=\frac{N I+E_{c o}+E_{p m}}{\left(R_{\text {air }}+R_{f e}+R_{c o}+R_{p m}\right) A}
\end{gathered}
$$

Electromagnetic force:

$$
\begin{gathered}
\mathrm{F}=\frac{d W_{a i r}}{d x}=B H A=\frac{B^{2} A}{\mu_{0}}=\frac{\phi^{2}}{\mu_{0} A} \\
=\frac{1}{\mu_{0} A}\left(\frac{N I+E_{c o}+E_{p m}}{R_{a i r}+R_{f e}+R_{c o}+R_{p m}}\right)^{2}
\end{gathered}
$$

The magnetic resistance :

$$
\begin{aligned}
& R_{\text {air }}=2 x / \mu_{0} A_{\text {air }} \\
& R_{f e}=L_{1} / \mu_{f e} A_{f e} \\
& R_{p m}=L_{2} / \mu_{p m} A_{p m} \\
& R_{c o}=L_{3} / \mu_{c o} A_{c o}
\end{aligned}
$$

\section{Under the typical levitation gap, analysis electromagnetic force}

\subsection{The selection of simulation conditions}

Maglev train has three typical load conditions, the no load in $23 \mathrm{t}$, heavy load in $31.5 \mathrm{t}$, full load in $35.5 \mathrm{t}$, and whole vehicle has 40 suspension electromagnet. A Maglev train has a total of 40 suspended electromagnet. Corresponding to each electromagnetic module, no load in $0.575 \mathrm{t}$, heavy load in $0.7875 \mathrm{t}$, full load in $0.8875 \mathrm{t}$. The electromagnetic characteristics of the suspended electromagnet in 3 typical operating conditions are analyzed, which are suspended in heavy load at gap $8 \mathrm{~mm}$, full load at gap $16 \mathrm{~mm}$, and no load at gap $3 \mathrm{~mm}$. Through the simulation of the rated suspension gap and floating gap, the two typical working conditions of the new type electromagnet are compared and analyzed. When the gap is relatively small, the traditional hybrid electromagnet in the suspension, will be adsorbed on the track, after power off, the electromagnet can not drop the case. So in the manufacture of mixed suspension electromagnet, two Ushaped iron core pole installed anti locking-track copper, in order to ensure minimum suspension air gap of $3 \mathrm{~mm}$. And in the case of no load, it is more likely to happen adsorption orbit fault. In the case of the gap $3 \mathrm{~mm}$ adsorption orbit, the aluminum nickel and cobalt reverse magnetization can be safely separated from the adsorption orbit state, to test whether it has the function of preventing the adsorption orbit.

\subsection{Simulation and analysis of electromagnetic force in suspension}

\subsubsection{Heavy load suspended at gap $8 \mathrm{~mm}$}

In the gap $8 \mathrm{~mm}$, the current from $0 \mathrm{~A}$ to $30 \mathrm{~A}$, the electromagnetic force vary with the change of the current situation, as shown in Fig. 3.

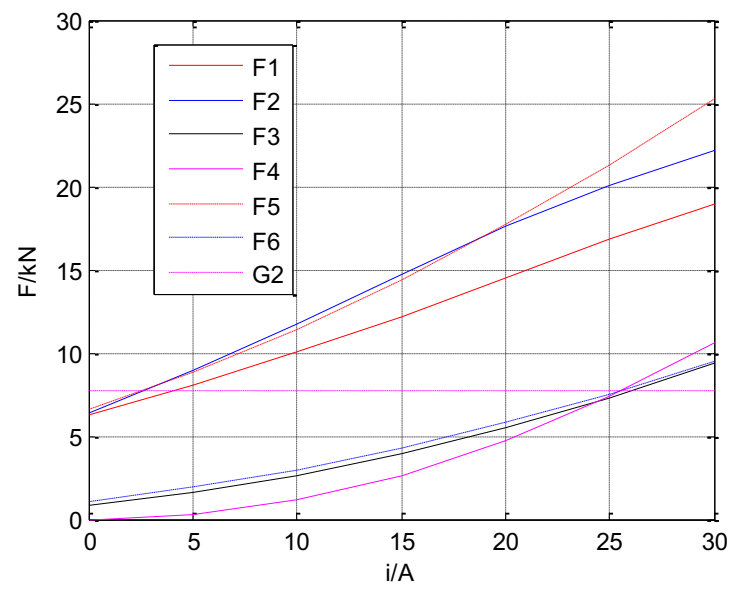

Figure 3. The electromagnetic force changes with the current situation at gap $8 \mathrm{~mm}$.

Notes: F1 is simulation value of electromagnetic force of $\mathrm{NdFeB}$ hybrid electromagnet; $\mathrm{F} 2$ is simulation value of electromagnetic force of AlNiCo (positive) hybrid electromagnet; F3 is simulation value of electromagnetic force of AlNiCo (negative) hybrid electromagnet; F4 is simulation value of electromagnetic force of electromagnetic magnet; F5 is theoretical calculation of electromagnetic force of AlNiCo (positive) mixed electromagnet; F6 is theoretical calculation of electromagnetic force of $\mathrm{AlNiCo}$ (negative) mixed electromagnet; $\mathrm{G} 2$ is the gravity of the electromagnet is under heavy load;

Can be seen from the figure,suspended in the gap $8 \mathrm{~mm}$ with heavy load, AlNiCo (positive) hybrid electromagnet suspension current is $3 \mathrm{~A}$ or so, slightly smaller than NdFeB hybrid electromagnet. From the electromagnetic force curve with the current changes, we can know, AlNiCo (positive) hybrid electromagnet and traditional $\mathrm{NdFeB}$ hybrid electromagnet has similar characteristics of suspension and curve slope for higher, performance is better than the traditional mixed electromagnet. From the graph, we can see that the curve of the simulation curve and the theoretical calculation of AlNiCo (positive) hybrid electromagnet is basically consistent with the curve. AlNiCo (positive) hybrid electromagnet has the same energy saving effect as the traditional mixed suspension electromagnet, and the control performance is better. 


\subsubsection{Full load suspended in gap $16 \mathrm{~mm}$}

Similarly in the $16 \mathrm{~mm}$ gap, to establish a current range from $0 \mathrm{~A}$ to $80 \mathrm{~A}$, the levitation force with the change of the current situation. As shown in Fig. 4.

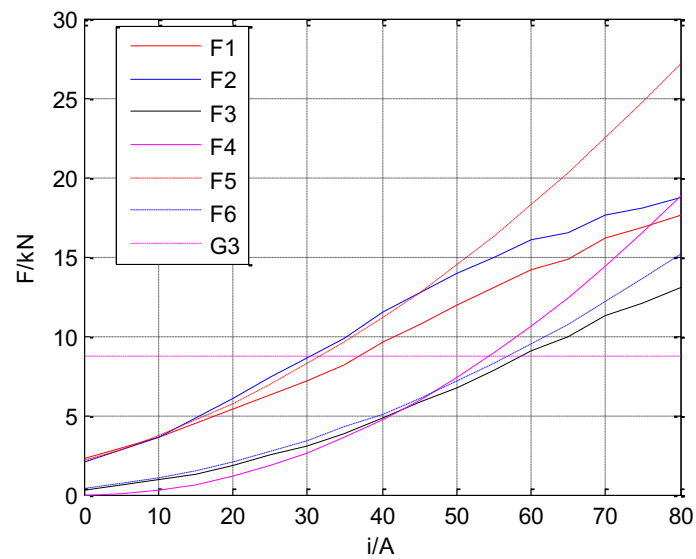

Figure 4. The electromagnetic force changes with the current situation at gap $16 \mathrm{~mm}$.

Notes: G3 is the gravity of the electromagnet is under full load;

When the full load of $16 \mathrm{~mm}$ is suspended, the electromagnetic electromagnet current reaches 52A, which almost reaches the saturation state. The traditional neodymium iron boron hybrid electromagnet current is $36 \mathrm{~A}$, and the floating current is relatively small. And AlNiCo (positive) hybrid electromagnet current is only $30 \mathrm{~A}$, the current is relatively low, and there is a lot of magnetic saturation allowance. It is in the middle of the electromagnetic force curve, the slope is big, the dynamic control performance is very good. Therefore, the aluminum nickel and cobalt mixed electromagnet is relatively small, the current load capacity of the circuit device can be relatively low, which is beneficial to simplify the power supply system and reduce the cost.

\subsubsection{No load suspended in gap $3 \mathrm{~mm}$}

Also at the gap $3 \mathrm{~mm}$, to establish a current range from $0 \mathrm{~A}$ to $30 \mathrm{~A}$, the levitation force with the change of the current situation. As shown in Fig. 5.

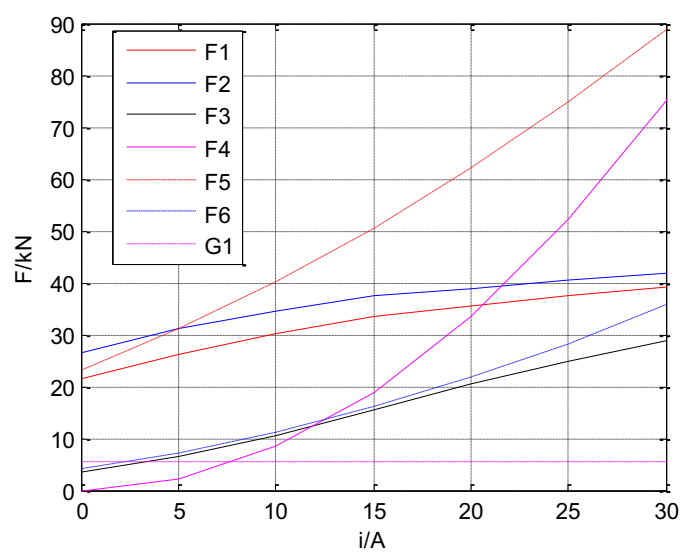

Figure 5. The electromagnetic force changes with the current situation at gap $3 \mathrm{~mm}$.
Notes: G1 is the gravity of the electromagnet is under no load;

No load at gap $3 \mathrm{~mm}$ is the position of the hybrid electromagnet which is easy to be adsorbed on the track. Usually, if the hybrid electromagnet is adsorbed on the track, then the reverse current is large, so that it falls off the track. But if this is the case, disconnect the power supply, hybrid electromagnet will lock rail, may damage the body. Therefore, at the gap $3 \mathrm{~mm}$, the investigation of the electromagnet in the 0 current situation, whether it has the ability to prevent the death of the dead, it is very important.

Can be obtained from Fig. 5, when the current is zero, $\mathrm{NdFeB}$ hybrid electromagnet electromagnet force about $22 \mathrm{kN}, \quad$ AlNiCo (positive) hybrid electromagnet electromagnetic force is about $26 \mathrm{kN}$, and the electromagnet in the no-load under the force of gravity for $5.635 \mathrm{kN}$, electromagnetic force is much larger than the gravity. So the hybrid electromagnets would lock the track. While AlNiCo (positive) hybrid electromagnet is adsorbed on the track, the pulse power supply through the reverse current around the aluminum nickel cobalt, so that the magnetic reverse, obtained AlNiCo (negative) hybrid electromagnet. And the excitation current is zero, the electromagnetic force of AlNiCo (negative) hybrid electromagnet is $3.44 \mathrm{kN}$, which is much less than the weight of the bearing. The electromagnet track will not be locked. Therefore, it has a good ability to prevent locking track. Parallel hybrid magnet and solenoid type electromagnet is almost as electromagnetic properties, not like NdFeB hybrid electromagnet that locking track.

\section{Conclusion}

In this paper, the theoretical derivation and Simulation of the new type of AlNiCo-NdFeB hybrid electromagnet are carried out. Confirmed the this structure can not only improve the electromagnetic force but also reducing the working current, and the aluminum nickel cobalt are reversely magnetized, thus achieving the goal of anti lock track.

Under the condition of heavy load at gap $8 \mathrm{~mm}$, excitation current of AlNiCo hybrid electromagnet, compared to solenoid type electromagnet, reduced about $750 \%$, that effectively reduces the excitation current of the electromagnet, so as to achieve the purpose of energy saving. At the rated operating gap, the slope of the current-electromagnetic force curve of AlNiCo hybrid electromagnet is larger than that of $\mathrm{NdFeB}$ hybrid electromagnet and the electromagnetic electromagnet, and the dynamic performance of AlNiCo hybrid electromagnet is optimal.

And under the condition of heavy load at gap $16 \mathrm{~mm}$, operating current of AlNiCo hybrid electromagnet is only about $83 \%$ of $\mathrm{NdFeB}$ hybrid electromagnet, and is about $57 \%$ of the electromagnetic magnet. AlNiCo hybrid electromagnet can greatly reduce the float current, and effectively reduces the power consumption of the system and installed capacity. Electromagnet volume, weight and the heat generation are also reduced. Thus the low-speed maglev train system cost and operation cost are reduced. 
At the same time, AlNiCo hybrid electromagnet can prevent locking track, and effectively overcome the security risks of traditional hybrid electromagnet locking rail. The new hybrid levitation electromagnet can effectively solve the problems of the electromagnet lock orbit, at the same time, have a good dynamic performance and suspension regulation performance. It has a very good application prospect.

\section{References}

1. Zhu Z Q, Chen Y S, Howe D. Maximising the fluxweakening capability of permanent magnet brushless AC machine and drives [C]. The Third International Power Electronics and Motion Control Conference, Beijing, China, (2000)
2. Morimoto S, Sanada M, Takeda Y. Performance of PM-assisted synchronous reluctance motor for highefficiency and wide constant-power operation[J]. IEEE Transactions on Industry Applications, (2001)

3. Wu Xiangming. Maglev train [M]. Shanghai: Shanghai Science and Technology Press(in Chinese), (2003)

4. Thornton R. The M3 Urban Transportation System[R]. MagneMotion Ine. America : Federal Transit Administration as Part ofFFA Project MA26-7077. (2003)

5. Xie Yunde. Modeling and simulation on EMS maglev vehicles [D]. Changsha: Natinal University of Defense Technology(in Chinese), (1998)

6. Ostovic V. Pole-changing permanent-magnet machines[J]. IEEE Trans. on Industry Applications, (2002) 